\title{
Coulomb breakup problem
}

\author{
A. S. Kadyrov, ${ }^{1}$ A. M. Mukhamedzhanov, I. Bray, ${ }^{1}$ and A. T. Stelbovics ${ }^{1}$ \\ ${ }^{1}$ Curtin University of Technology, GPO Box U1987, Perth, WA 6845, Australia
}

Scattering in a few-body system is one of the central subjects of quantum mechanics. However, conventional scattering theory is formally valid only when the particles interact via short-range potentials. For charged particles with the long-range Coulomb interactions the theory requires modification. In the time-dependent formulation, formal scattering theory is generalized to include Coulomb potentials by choosing appropriately modified time evolution operators. This is equivalent to various forms of screening and renormalization in the time independent formulation. The renormalization method leads to the correct cross sections for the two-body problem; however, the results from this procedure cannot be regarded as completely satisfactory. For instance, different ways of screening lead to different asymptotic forms for the scattering wave function. Recently we have demonstrated that there was a general approach to the two body collision problem [1] that did not lead to the aforementioned formal difficulties and did not require renormalization. The situation in a few-body system is even more complicated. Rigorous scattering theory for a system of three particles valid for short-range potentials was given by Faddeev [2]. A renormalization method was implemented for the three-body problem when only two particles are charged [3].

In this work [4] we present a surface-integral approach to formulating scattering theory. We use the recently derived analytic results for total scattering wave functions in asymptotic domains to develop a well-defined prior and post forms of the breakup amplitude valid for short-range and Coulomb potentials. These new definitions do not require screening and renormalization or any regularization as they are exact and divergence-free. We have derived for the first time post and prior forms of the breakup amplitude for a three-body system that are valid for both short-range and Coulomb potentials. This was made possible by the recently obtained analytic forms of the asymptotic wave functions combined with a surface integral approach to the scattering theory. This work published in Phys. Rev. Lett. 101, 230405 (2008).

[1] A. S. Kadyrov, I. Bray, A. M. Mukhamedzhanov, and A. T. Stelbovics, Phys. Rev. A 72, 032712 (2005).

[2] L. D. Faddeev, Tr. Matem. Inst. V. A. Steklova, Akad. Nauk. SSSR 69, 1 (1963).

[3] E. O. Alt, W. Sandhas, and H. Ziegelmann, Phys. Rev. C 17, 1981 (1978).

[4] A. S. Kadyrov, A. M. Mukhamedzhanov, I. Bray, and A. T. Stelbovics, Phys. Rev. Lett. 101, 230405 (2008). 